\title{
Parametrical Study of Free Vibrati- on and Plastic Collapse of a Clamped Beam
}

Pavel Élesztős (SK) pavel.elesztos@stuba.sk

Ladislav Écsi (SK) ladislav.ecsi@stuba.sk

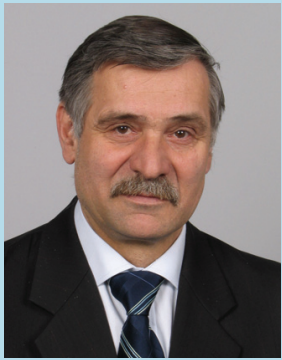

\section{BIOGRAPHICAL NOTES}

prof. Ing. Pavel Élesztős, CSc. was born on 6th May 1948. He graduated from the Faculty of Mechanical Engineering at the Slovak University of Technology in Bratislava in 1972, where he defended his Ph.D. thesis in 1977. He became an associate professor of mechanics of solid pliable bodies and mediums in 1988, which field remained his main research area. He was awarded another associate professor title in 2007 and was nominated full professor of applied mechanics in 2010. From 1997 until 2006 he acted as the head of The Department of Elasticity and Strength of Materials and soon after, in 2007, he became the deputy head of the Institute of Applied Mechanics and Mechatronics of the STU in Bratislava. He is a member of the Slovak Society for Mechanics and the Central European Associations for Computational Mechanics. His research area includes heat problems and associated stress fields of granulate materials during their technological processing. He proposed an original method for the analytical solution of temperature and stress fields of co-current and contra-current heat exchangers using a liquid coolant and a granulate medium. He carried out numerous finite element analyses of various technological processes such as welding processes and their optimization employing genetic algorithms and achieved outstanding results in solving non-standard problems of nuclear power plants, as well as the chemical and the oil and gas industry.

\section{KEY WORDS}

FEM, Updated Lagrangian Formulation, Von Mises Material Model, Multilinear Isotropic Hardening, Internal Damping

\section{ABSTRACT}

In this paper a parametrical study of free vibration and plastic collapse of a clamped beam is presented. In the numerical study the finite element method was used utilising the updated Lagrangian formulation for large deformations/large strains, the Von Mises material model with multilinear isotropic hardening for hypoelastic-plastic materials, the Kelvin-Voight's model for internal damping and the Jaumann's objective rate in the form of the Green-Naghdi's objective rate for co-rotational Cauchy's stress update calculation, in which appropriate rotation tensors were expressed with the Rodrigues' formula. The aim of this study was to numerically determine the range of the material parameters of the Kelvin-Voight's damping model using a real material, such as low carbon steel, which 
is a commonly used construction material in contemporary engineering practice. In the numerical experiments elastic and plastic loading cases were studied applying pressure on the upper surface of the bar free end as stepped load using the Heaviside step function. A few relevant results of the study are presented and briefly discussed too.

\section{INTRODUCTION}

An engineering construction during its operation has to withstand various loadings; the majority of which can be characterized as dynamic or cyclic. To model properly the behaviour of such a construction internal/material damping of the construction material has to be taken into account. Although technical literature differentiates between material or structural viscous damping, nonmaterial viscous damping, Coulomb or dry damping and other nonmaterial damping models [1], the material damping is merely an issue of rate dependent models of viscoelasticity or viscoplasticity which have been developed to simulate materials with memory, creep or relaxation using convolution integrals [2-4]. The Rayleigh damping, or in other words the proportional damping, is the most widely used damping model in contemporary dynamic analyses [5-8], however it has to be carefully used in continuum based models, as the damping model does not differentiate between rigid body motion and deformation, which might lead to spurious numerical behaviour and inaccurate results. This applies mainly in such cases where high speed rigid body motion takes place during the deformation of the body.

\section{THEORY BACGROUND}

In the problem formulation the updated Lagrange method [9] for large strains/large deformations is used. The material of a solid deformable body of volume $\Omega$ and surface $\partial \Omega$ is idealised as non-polar continuum [10]. The equation of motion, defined with the conservation of linear momentum of the body, is given in the following variational form [11]:

$$
\int_{\Omega} \rho \dot{\mathbf{v}} \cdot \delta \mathbf{v} d v+\int_{\Omega} \sigma: \delta \mathbf{d} d v=\int_{\Omega} \mathbf{b} \cdot \delta \mathbf{v} d v+\int_{\partial \Omega} \mathbf{t} \cdot \delta \mathbf{v} d s,(1)
$$

In equation (1) $\rho, \mathbf{v}, \sigma, \mathbf{d}, \mathbf{b}, \mathbf{t}, \mathbf{f}_{i}$ denote the material density, the velocity vector, the Cauchy stress tensor, the strain rate tensor, the body force vector, the surface traction vector and the nodal force vector

respectively at a material point of the body. Considering the co-rotational formulation [9] adjustment of the Kelvin-Voigt model for [12] for finite strain elastoplasticity [13] results in the co-rotational Cauchy's stress tensor $\hat{\sigma}=\mathbf{R}^{T} \cdot \sigma \cdot \mathbf{R}$, eqn. (2) to be split into two objective stress measures, represented by an incrementally changing elastic part or corotational elastic Cauchy's stress tensor $\hat{\sigma}^{\text {el }}$, eqn. (3), and an instantaneous damping part or co-rotational viscous Cauchy's stress tensor $\hat{\sigma}^{\text {damp }}$, eqn. (6), where the co-rotational elastic Cauchy's stress increment $\Delta \hat{\sigma}^{e l}$ is defined either with eqn. (4) in elastic loading/ unloading or with eqn. (5) in plastic loading.:

$$
{ }^{n+1} \hat{\boldsymbol{\sigma}}={ }^{n+1} \hat{\boldsymbol{\sigma}}^{e l}+{ }^{n+1} \hat{\boldsymbol{\sigma}}^{\text {damp }},
$$

where

$$
\begin{aligned}
& { }^{n+1} \hat{\boldsymbol{\sigma}}^{e l}=\Delta \hat{\boldsymbol{\sigma}}^{e l}+{ }^{n} \hat{\boldsymbol{\sigma}}^{e l}, \\
& \Delta \hat{\boldsymbol{\sigma}}^{e l}=\Delta t^{n+\frac{1}{2}} \hat{C}^{\nabla J}:{ }^{n+\frac{1}{2}} \hat{\mathbf{d}} \text {, in el.load. / unload } . \\
& \Delta \hat{\boldsymbol{\sigma}}^{e l}=\Delta t^{n+\frac{1}{2}} \hat{C}^{\nabla J}:\left({ }^{n+\frac{1}{2}} \hat{\mathbf{d}}-{ }^{n+\frac{1}{2}} \hat{\mathbf{d}}^{p l}\right) \text {, in pl.load. } \\
& { }^{n+1} \hat{\boldsymbol{\sigma}}^{\text {damp }}={ }^{n+1} \hat{C}^{\nabla J d a m p}:{ }^{n+1} \hat{\mathbf{d}}, \\
& { }^{\circ} C^{\nabla J}=2 G \mathrm{I}+\lambda(\mathbf{1} \otimes \mathbf{1}), \\
& G=\frac{E}{2(1+v)}, \\
& \lambda=\frac{v E}{(1+v)(1-2 v)}, \\
& n+\frac{1}{2}{ }_{a b c d}^{\nabla}= \\
& \frac{{ }^{n+\frac{1}{2}} R_{m a}{ }^{n+\frac{1}{2}} R_{n b}{ }^{n+\frac{1}{2}} R_{o c}{ }^{n+\frac{1}{2}} R_{p d}{ }^{n+\frac{1}{2}} F_{m i}{ }^{n+\frac{1}{2}} F_{n j}{ }^{n+\frac{1}{2}} F_{o k}{ }^{n+\frac{1}{2}} F_{p l}{ }^{0} \quad{ }_{i j k l} J}{n+\frac{1}{2} J},(10) \\
& { }^{\circ} C^{\text {vidanp }}=2 G^{\text {danp }} \mathrm{I}+\lambda^{\text {danp }}(\mathbf{1} \otimes \mathbf{1}), \\
& G^{\text {danp }}=\frac{E^{\text {danp }}}{2\left(1+v^{\text {danp }}\right)}, \\
& \lambda^{\text {dump }}=\frac{v^{\text {damp }} E^{\text {danp }}}{\left(1+v^{d \omega n p}\right)\left(1-2 v^{d a m p}\right)}, \\
& { }^{n+1} \hat{C}_{\text {abcd }}^{\text {Vdamp }}= \\
& \frac{{ }^{n+1} R_{m a}{ }^{n+1} R_{n b}{ }^{n+1} R_{o c}{ }^{n+1} R_{p d}{ }^{n+1} F_{m i}{ }^{n+1} F_{n j}{ }^{n+1} F_{o k}{ }^{n+1} F_{p l}{ }^{0} \mathrm{C}_{i j k l}^{\text {VJdamp }}}{{ }^{n+1} J},
\end{aligned}
$$


In the co-rotational elastic Cauchy's stress integration, eqn. (3), the midpoint rule and the Jaumann's objective rate are used, which can also be expressed in an equivalent Green-Naghdi's objective rate form, if the corresponding rotation tensors take the following forms $[14,15]$

$$
\begin{aligned}
& { }^{n+\frac{1}{2}} \mathbf{R}=\exp \left[\frac{\Delta t}{2}{ }^{n+\frac{1}{2}} \mathbf{W}\right] \cdot{ }^{n} \mathbf{R}, \\
& { }^{n+1} \mathbf{R}=\exp \left[\Delta t^{n+\frac{1}{2}} \mathbf{W}\right] \cdot{ }^{n} \mathbf{R},
\end{aligned}
$$

where $\mathbf{W}$ stands for the spin tensor. In the tensor exponential function (15) and (16) calculation the Rodrugues' formula [15] was used. No objective integration is needed in the co-rotational viscous Cauchy's stress tensor calculation (6), as the stress tensor is objective and is instantaneous in its nature. In eqns. (2)-(16) and (17)-(19) the left superscripts $n, n+1 / 2, n+1$ denote the physical quantity value at discrete times, corresponding to previous, mid and current configuration of the body within the current time step $\Delta t$. We assume that the material in the initial configuration of the body is isotropic, and that the co-rotational strain rate tensor $\hat{\mathbf{d}}=\mathbf{R}^{T}$. $\mathbf{d} \cdot \mathbf{R}$ has the additive decomposition $\hat{\mathbf{d}}=\hat{\mathbf{d}}^{e l}+\hat{\mathbf{d}}^{p l}$ into an elastic part $\hat{\mathbf{d}}^{e l}$ and a plastic part $\hat{\mathbf{d}}^{p l}$. In this work the St. Venant-Kirchov's material model is used [11], the elastic material tensor ${ }^{0} \mathrm{C}^{\nabla J}$ of which can be expressed with eqns. (7)-(9) in the initial configuration. Here $G$ and $\lambda$ denote the shear modulus and the Lame's constant, I,I stand for a fourth-order unit tensor and a second-order unit tensor and $\nu, E$ are respectively the Poisson's ratio and the Young's modulus. The fourth-order damping tensor ${ }^{0} \mathrm{C}^{\nabla \text { Jdamp }}$ is formally constructed in the same way, eqns. (11)(13) in the initial configuration as the elastic material tensor using two independent variables $\nu^{\text {damp }}, E^{\text {damp }}$, which ensures isotropy. The advantage of such a formulation is that it enables easy experimental identification of the material parameters using amplitude measurements of thin-walled tubes in free, longitudinal and torsional vibration. In order to use the fourth-order elastic and damping material tensor for stress update calculation, they have to be transformed into the mid and current configuration within the current time step and subsequently rotated into the reference configuration for stress update calculation [9] using eqns. (10) and (14), in which $\mathbf{F}$ stands for the deformation gradient and $J=\operatorname{det}(\mathbf{F})$ is the Jacobian or the determinant of $\mathbf{F}$ respectively. Eqns. (2)-(16) are supplemented with the following constitutive and evolution equations:

$$
\begin{gathered}
{ }^{n+1} f={ }^{n+1} \sigma_{e q}-{ }^{n+1} \sigma_{y} \leq 0, \\
{ }^{n+1} \sigma_{e q}=\sqrt{\frac{3}{2}{ }^{n+1} \hat{\boldsymbol{\Sigma}}:{ }^{n+1} \hat{\boldsymbol{\Sigma}}}, \\
{ }^{n+1} \sigma_{y}={ }^{n+1} H \cdot \Delta \varepsilon^{p l}+{ }^{n} \sigma_{y} .
\end{gathered}
$$

Equations (17), (18) define the Von-Mises yield surface in terms of the deviatoric part of the co-rotational Cauchy's stress tensor $\boldsymbol{\Sigma}$ and the material current yield stress $\sigma_{y}$. In this work associative plasticity is used with multilinear isotropic hardening Eqn. (19), where the variables ${ }^{n+1} H, \Delta \varepsilon^{p l}$ denote the material current hardening modulus and the accumulated plastic strain increment.

\section{NUMERICAL EXAMPLE}

In the numerical study free vibration and plastic collapse of a cantilever beam, length $600 \mathrm{~mm}$ and rectangular cross section and size $50 \mathrm{~mm} \times 50 \mathrm{~mm}$ is studied using elastic and plastic loading. In the parametrical study internal damping of the material is varied applying pressure on the upper surface of the free end of the beam as stepped load using the Heaviside step function. Figure 1 depicts the spatially discretized model, the boundary conditions and the applied pressure with arrows

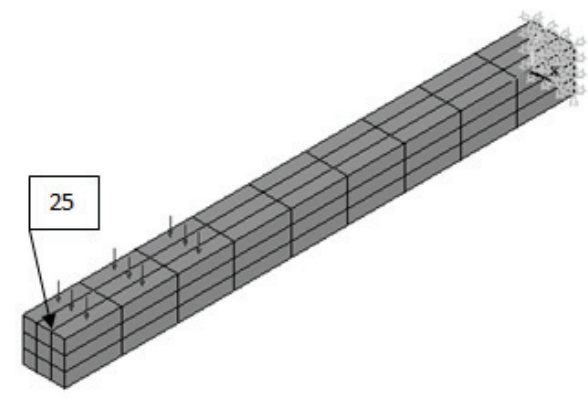

\section{Fig. 1 Spatially discretized model}

In the numerical experiments we assume, that the cantilever beam is manufactured from low carbon steel with constant material properties. Table 1 lists the material properties of the model. 


\begin{tabular}{|l|l|}
\hline$\rho\left[\mathrm{kg} \cdot \mathrm{m}^{-3}\right]$ & 7800,0 \\
\hline$E[\mathrm{GPa}]$ & 210,0 \\
\hline$E^{\text {damp }}[\mathrm{Pa} . \mathrm{s}]$ & $0,2.1 .10^{4} ; 2,1.10^{5} ; 2,1.10^{6} ;$ \\
& $2,1.10^{7} ; 2,1.10^{8} ; 4,2.10^{8}$ \\
\hline$\nu[-]$ & 0,3 \\
\hline$\nu^{\text {damp }}[-]$ & 0,3 \\
\hline$\sigma_{y}[\mathrm{MPa}]$ & 245,0 \\
\hline
\end{tabular}

Tab. 1 Material properties

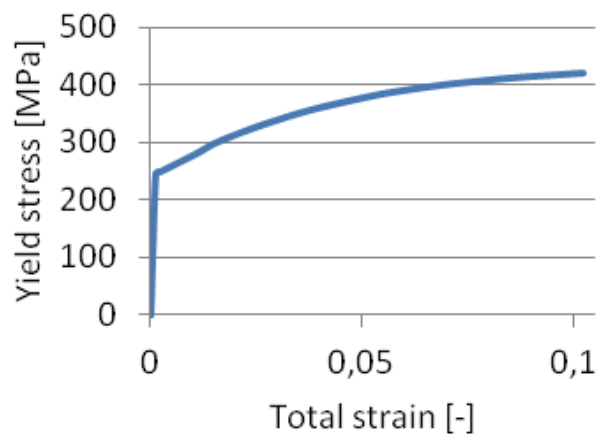

Fig. 2 Material stress-strain curve

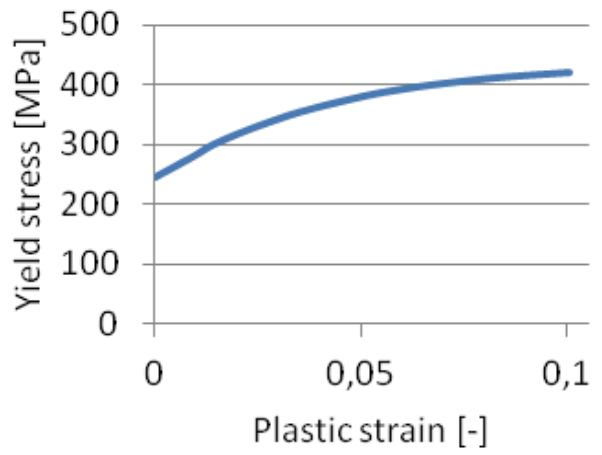

Fig. 3 Material stress-strain curve in terms of plastic strain

Figures 2 and 3 show the material stress-strain curves in term of total/plastic strain for stress update calculation. The hardening modulus ${ }^{n+1} H$ for multilinear isotropic hardening in eqn. (19) can be determined from Fig. 3. In the numerical simulations 8 node solid elements with linear shape functions are used. The analyses have been run as dynamic ones with implicit time integration employing the trapezoidal rule. In the numerical simulations constant time step, size $\Delta t=1,0.10^{-4}$ is used. Elastic and plastic loading cases have been studied applying/ disregarding the internal/material damping. In the elastic case free vibration of the cantilever beam is investigated, applying a constant $p=0,9$ MPa pressure at the beam free end. In the plastic case the beam collapse is examined by simply increasing the pressure value to $p=5,0 \mathrm{MPa}$. The other parameters of the model have been kept unchanged.

\section{NUMERICAL RESULTS}

Figure 4 shows the vertical deformation time history curves for various internal damping at node 25 on the free end of the beam during free vibration using elastic loading. (see. Fig. 1 for the location of the node N25) As it can be seen in the figure, the internal damping is an important material parameter which dissipates energy and results in the beam to reach the static equilibrium in a few cycles, depending on the exact value of the material parameter. Zero damping means no mechanical energy dissipation and conservative system. The damping values $E^{\text {damp }}$ between zero and $2,1.10^{4} \mathrm{~Pa}$.s are considered to be low and almost unrealistic.

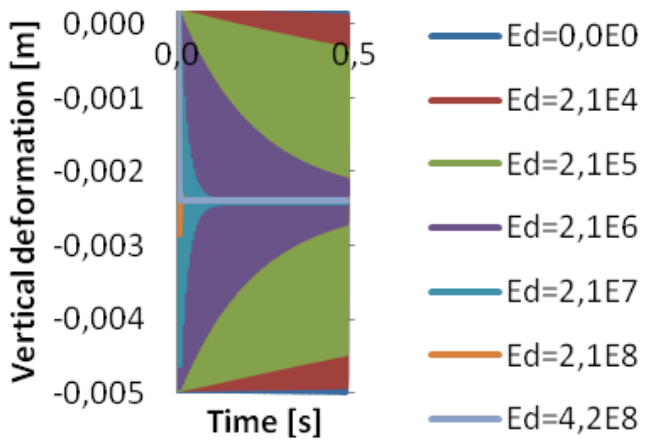

Fig. 4 Vertical deformation time history at node 25 using elastic loading

The internal damping $E^{\text {damp }}$ of a real material (low carbon steel) lies between $2,1.10^{4} \mathrm{~Pa}$.s and 2,1.10 Pa.s The damping values above $2,1.10^{7}$ Pa.s are considered to be high, with exponential course of the time history curve above $4,2.10^{8}$ Pa.s. Figures 5-7 depict a few selected results at the end of the plastic collapse of the cantilever beam using $E^{\text {damp }}=$ $2,1.10^{7} \mathrm{~Pa}$.s internal damping. They are the vertical deformation, the Von Mises stress distribution and the accumulated plastic strain distribution over the body at the end of the numerical analysis.

In order to make further investigation vertical deformation time history curves are plotted for different 
internal damping values at node 25 of the beam. The corresponding results are shown in figure 8. Again we can conclude that the internal damping is an important material property, which not only influences how long the body vibrates after excitation but also affects the plastic deformations, that is the higher the internal damping value, the lower the amount of the plastic deformation at a material point of the body. Moreover it is obvious from the figure that even for very high damping values, where the time history curves become exponential with no oscillations in them, the plastic collapse of the cantilever beam takes place in a very short time, just above $0,1 \mathrm{~s}$. Such behaviour is due to the fact, that the Kelvin-Voigt model is independent of rigid body motion. The collapse would take much longer or even unreasonably longer, if the Rayleigh's damping was to be used, as the model depends on rigid body motion, which makes it inappropriate to model internal damping.
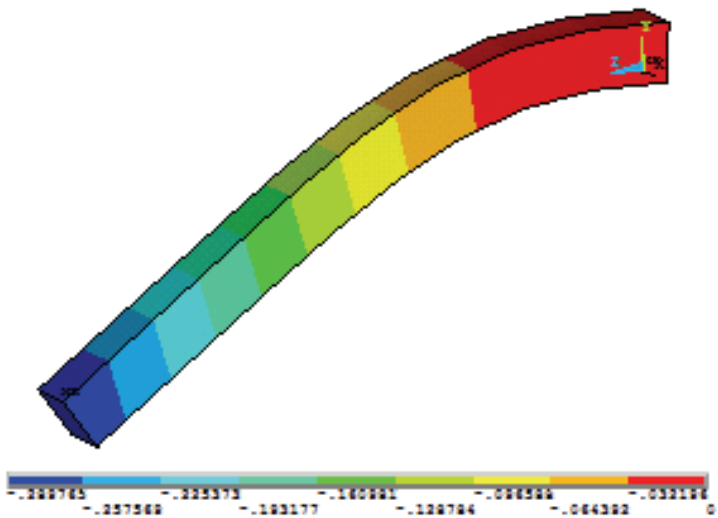

Fig. 5 Vertical deformation of the beam at the end of the plastic collapse $E^{\text {damp }}=2,1.10^{7}$

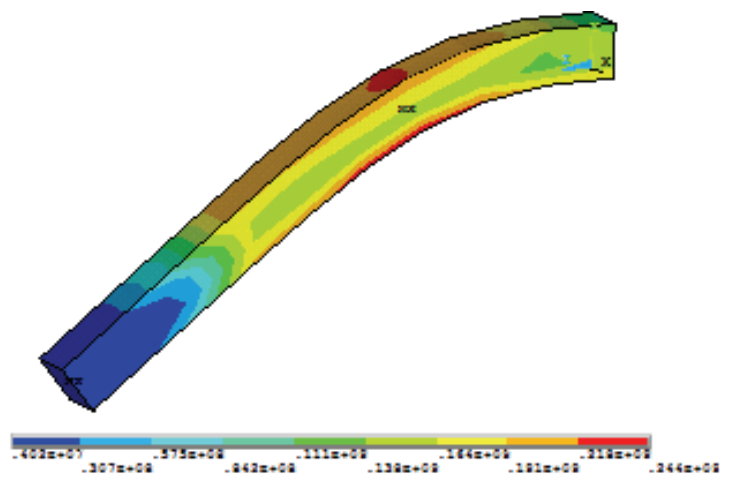

Fig. 6 Von Mises stress distribution at the end of the plastic collapse $E^{\text {damp }}=2,1.10^{7}$

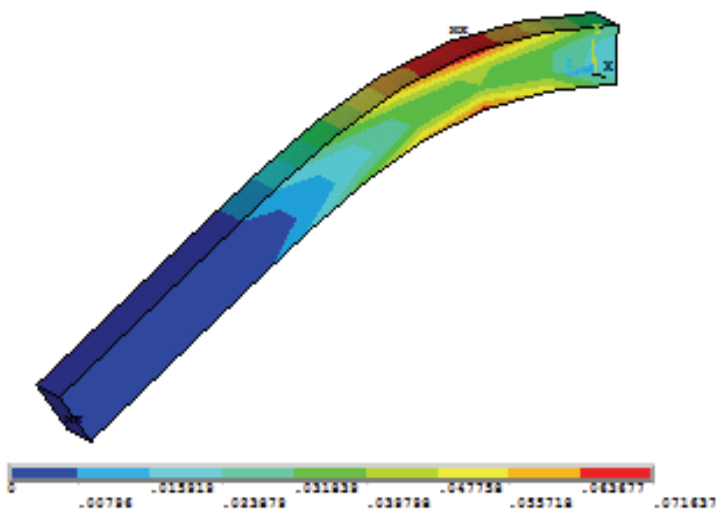

Fig. 7 Accumulated plastic strain distribution at the end of the plastic collapse $E^{\text {damp }}=2,1.10^{7}$

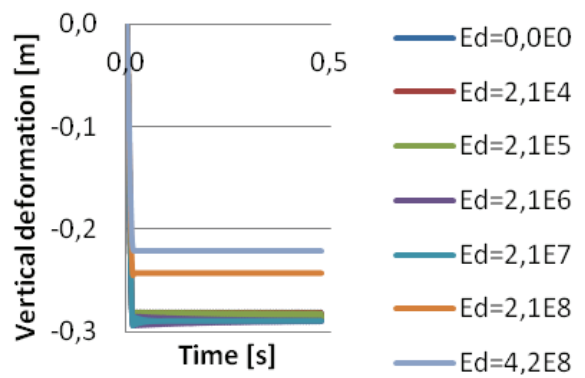

Fig. 7 Vertical deformation time history at node 25 using elastic-plastic loading

\section{CONCLUSION}

In this paper a universal constitutive equation using the Kelvin-Voigt model for internal damping and the updated Lagrangian formulation for large deformations/large strains are presented. Free vibration and plastic collapse of the cantilever beam have been studied using various damping values. The range of low, normal and high internal damping of low carbon steel has been determined. The model is capable of simulating real material behaviour under cyclic and dynamic loading more realistically as is independent of rigid body motion. This significantly improves the accuracy of the numerical analyses, where high speed rigid body motion or deformations at high strain rates take place.

\section{ACKNOWLEDGEMENT}

Funding using the VEGA grant 1/0488/09 and $1 / 0051 / 10$ resources is greatly appreciated. 


\section{REFERENCES}

[1] C.T. Sun, Y.P. Lu, Vibration damping of structural elements, Prentice Hall PTR., Englewood Cliffs, New Jersey, 1995

[2] J. Lemaitre, Handbook of Material Behavior Models, Vol. 1, Deformations of Materials, Academic Press, London, 2001

[3] Cs. Asszonyi et all., Izotrop kontinuumok anyagtulajdonságai, Múegyetemi kiadó, Budapest, 2008

[4] A.D. Mesquita, H.B. Coda, Alternative Kelvin viscoelastic procedure for finite elements, Appl. Math. Model., no. 26, 2002, pp. 501-516

[5] J. Argiris, H.P. Mlejnek, Dynamics of structures, Elsevier Science Publishers, Amsterdam, 1991

[6] K.J. Bathe, Finite element procedures, Prentice-Hall INC., Englewood Cliffs, New Jersey, 1995

[7] U. Lee, Spectral element method in structural Dynamics, John Wiley \& Sons Pte Ltd., 2009

[8] M. Liu, D.G. Gorman, Formulation of Rayleigh damping and its extensions, Comput. \& Struct., vol. 57, no. 2, 1995, pp. 277-285

[9] T. Belytschko, W.K. Liu, B. Moran, Nonlinear finite elements for continua and structures, John Wiley \& Sons LTD, Chichester, 2000

[10] G.A. Holzapfel, Nonlinear solid mechanics, A continuum approach for engineering, John Wiley \& Sons LTD., Chichester, 2001

[11] J. Bonet, R.D. Wood, Nonlinear continuum mechanics for finite element analysis, Cambridge University Press, Cambridge, 1997

[12] L. Écsi, P. Élesztős, Constitutive equation with internal damping for materials under dynamic and cyclic loadings, 46th International conference in Experimental Stress Analysis 2008, EAN 2008, Horní Bečva, ČR, 2.-5. 6. 2008

[13] L. Écsi, P. Élesztős, J. Kosnáč, Constitutive equation with internal damping for materials under cyclic and dynamic loadings using large strain/large deformation formulation, The International Conference on Computational Modelling and Advanced Simulations 2009, CMAS 2009, Bratislava, Slovak Republic, 2009

[14] J. C. SIMO, T.J.R. HUGHES, Computational Inelasticity, Springer, New York, 1998

[15] E.A. de Souza Neto, D. Perić, D.R.J. Owen, Computational Methods for Plasticity, Theory and Applications, John Wiley \& Sons Ltd., Singapore, 2008

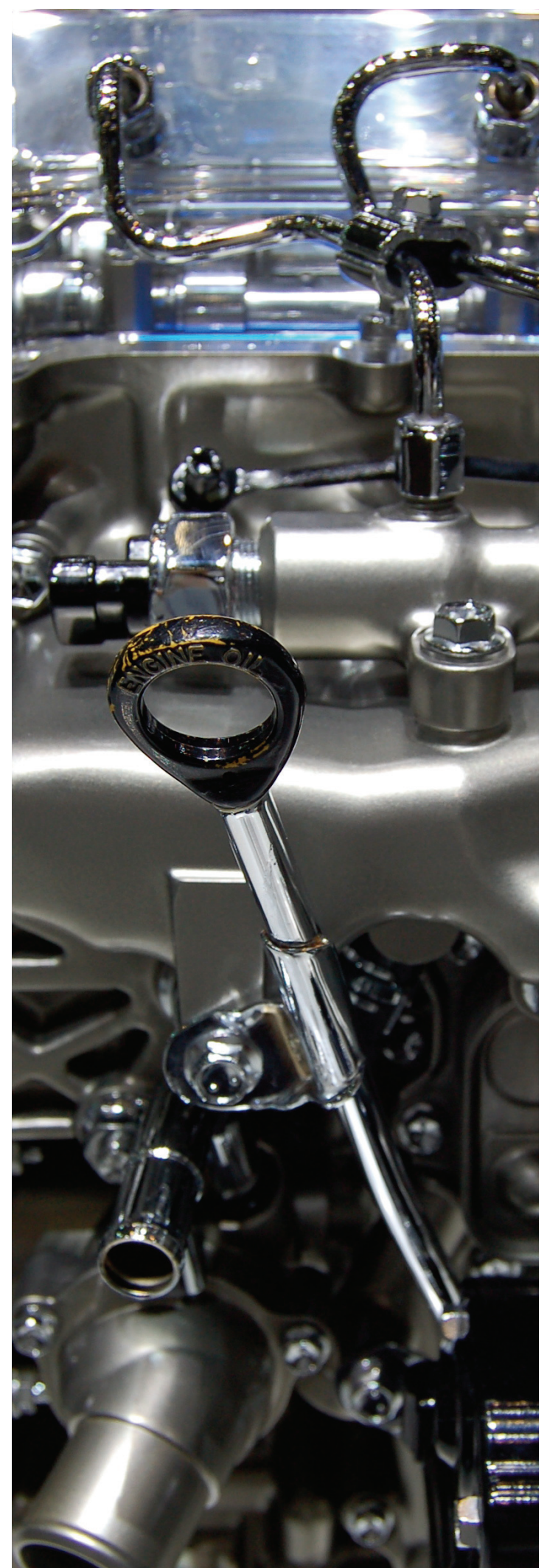

